\title{
Efficacy of Danshen (Salvia miltiorrhiza) Dripping Pills for Prevention of Acute Mountain Sickness: A Randomized, Double-Blind, Placebo-Controlled Trial
}

\author{
Kui Li ${ }^{1}$, Lijun Li', Jialun Fu ${ }^{2}$, Yi He², Zhaohui Song ${ }^{2}$, Shuiping Zhou ${ }^{2}$, \\ Luobu Gesang ${ }^{1 *}$ and Rui Liu ${ }^{2 *}$ \\ ${ }^{1}$ Tibet Autonomous Region People's Hospital, Tibet Institute of High Altitude Medicine, \\ Lhasa, China \\ ${ }^{2}$ Tasly Pharmaceutical Group Company Limited, Tianjin, China
}

\begin{abstract}
A B S T R A C T
A randomized, double-blind, placebo-controlled trial was conducted to investigate the efficacy of prophylaxis by administering compound Danshen dripping pills (CDDPs) on acute mountain sickness (AMS). To this end, a total number of 58 healthy low-altitude inhabitants residing in Beijing were randomized to two treatment sequences, receiving either $270 \mathrm{mg}$ CDDPs or placebo during an 11-day trip in Tibet, China, at an altitude of more than $3000 \mathrm{~m}$. The Lake Louise Scoring System (LLSS) was also utilized to access AMS incidence and severity, twice a day, during the research period. AMS was further diagnosed when headaches and LLSS $\geq 3$ were present. A total of 54 subjects completed the study and no significant difference was observed in the number of individuals with AMS between the groups. However, during the whole observation period, the LLSS values for comprehensive AMS symptoms in the group receiving CDDPs indicated lower occurrence than those in the placebo (CON) group. It is noteworthy that, on day 3 (arrival at Lhasa, altitude $3658 \mathrm{~m}$ ), AMS symptoms (LLSS=3.79) in the CON group had clearly aggravated $(\mathrm{P}=0.0475)$ in contrast to those in the group with administration of CDDPs (LLSS $=2.31)$. Besides, arterial oxygen saturation $\left(\mathrm{SaO}_{2}\right)$ value for those taking CDDPs was significantly higher than that in the CON group $(87.86 \%$ vs. $85.72 \%, \mathrm{P}=0.0388)$ on day 10 . These preliminary findings suggested that CDDPs might be regarded as an effective prophylaxis for AMS to a certain extent without any specific adverse effects, relieving clinical manifestations of AMS, especially after rapid ascents (Clinical Trial Registry Number: NCT03270787).
\end{abstract}

\begin{tabular}{l} 
Article Information \\
Received 04 December 2019 \\
Revised 02 March 2020 \\
Accepted 13 April 2020 \\
Available online 04 September 2020 \\
Authors' Contribution \\
\hline RL and LG conceived and designed \\
the study. RL, LG, JF, YH, ZS and \\
SZ contributed to the preparation \\
of the manuscript. LG, KL, LL, \\
RL performed the trial. JF, YH, ZS \\
prepared Figures 1-4. KL, LL, JF, YH \\
and SZ prepared Tables and Figures \\
S1-S3. All authors also read and \\
approved the manuscript. \\
Key words \\
\hline Acute Mountain Sickness (AMS), \\
Compound Danshen (Salvia \\
miltiorrhiza) Dripping Pills (CDDPs), \\
Lake Louise Scoring System (LLSS), \\
Unacelimatized Individuals, High \\
Altitude
\end{tabular}

\section{INTRODUCTION}

$\mathrm{A}$ cute mountain sickness (AMS) often occurs in lowaltitude residents or unacclimatized individuals who ascend rapidly to regions with above $2500 \mathrm{~m}$ altitude (Luks et al., 2017). More than one-fourth of people travelling to above $3500 \mathrm{~m}$ and more than one-half of those traveling to above $6000 \mathrm{~m}$ attitudes are also likely to suffer from AMS, whose symptoms include headaches, fatigue, dizziness, nausea, sleepiness, insomnia, and heart palpitations that commonly appear 6 to $12 \mathrm{~h}$ after ascents and range from mild to severe (Jin, 2017). If not treated promptly, AMS can progress to high-altitude cerebral edema (HACE) or high-altitude pulmonary edema (HAPE) under sustained hypoxia, which are potentially fatal (Jafarian et al., 2010). Likewise, AMS is known as a pathophysiological syndrome caused by rapid ascents to higher altitudes

\footnotetext{
Corresponding author: liuruitj2008@126.com; gesang5354@sina.com 0030-9923/2020/0006-2215 \$9.00/0

Copyright 2020 Zoological Society of Pakistan
}

without acclimatization (Hackett et al., 2001), whose incidence has been reported to be proportional to ascent rates. For unacclimatized individuals who have rapidly ascended to altitudes of $4559 \mathrm{~m}$, the incidence of AMS has been by 58\% (Carod-Artal, 2014; Schneider et al., 2002) and at least $25 \%$ of cases have experienced headaches at altitudes above $2000 \mathrm{~m}$. However, according to the International Headache Society (IHS), the incidence rate of high-altitude headaches increases to $80 \%$ at altitudes above $3000 \mathrm{~m}$ for individuals with no pre-exposure, and can even reach $100 \%$ at $4500 \mathrm{~m}$ or higher (Wilson et al., 2009). Prophylactic treatment by rational administration of drugs can thus prevent or alleviate symptoms of AMS. Accordingly, appropriate drugs include acetazolamide

\section{Abbreviations}

AMS, acute mountain sickness; BMI, body mass indices; CDDPs, compound Danshen (Salvia miltiorrhiza) dripping pills; CHD, coronary heart disease; CI, confidence interval; HACE, high- altitude cerebral edema; HAPE, high-altitude pulmonary edema; ISMM, International Society for Mountain Medicine; LLSS, Lake Louise Scoring System; $\mathrm{SaO}_{2}$, blood oxygen saturation; TCM, Traditional Chinese medicine. 
(Kayser et al., 2012), non-steroidal anti-inflammatory drugs (NSAIDS) (such as acetaminophen, ibuprofen, etc.) (Pandit et al., 2014), and dexamethasone (Tang et al., 2014), among which, only $70 \%$ of the cases would suggest acetazolamide and $50 \%$ of them would prefer paracetamol for medical treatment of AMS (Métrailler et al., 2019). The frequently documented side effects of these conventional drugs, including paresthesia, dysgeusia, diuresis, myasthenia, and gastrointestinal discomfort, must be considered prior to prescriptions (Basnyat et al., 2006). Traditional Chinese medicine (TCM) is also becoming increasingly recognized and accepted, both in China and abroad, largely due to its advantages of fewer side effects, facile administration, and effective protection of target organs. Over the last 20 years, compound Danshen (Salvia miltiorrhiza) dripping pills (CDDPs) have become widely advocated as an effective treatment for angina and coronary heart disease (CAD) (Luo et al., 2015). CDDPs contain Salvia miltiorrhiza, Radix notoginseng, and Borneolum syntheticum, which have beneficial synergistic effects on blood circulation (or removing blood stasis) as well as "clearing away heat-evil" and relieving pain (O'Brien et al., 2011). Significantly, CDDPs were the first proprietary TCM that could successfully complete a phase-II clinical trial in the United States, in which they were employed for the treatment of chronic stable angina, and had been approved by the Australian Therapeutic Goods Administration (Yang et al., 2014). On the basis of the studies cited above, it was reasoned that CDDPs might be an effective medicine for prevention and treatment of AMS through relaxation of cerebral vascular tissues, improvement of microcirculation, and relief of other symptoms associated with hypoxia.

In view of that, a randomized, double-blind, placebocontrolled clinical trial was conducted to investigate the efficacy of CDDPs as an antihypoxiant to alleviate AMS symptoms. Like most other clinical trials on AMS prevention using prophylactic herbal medications (Tsai et al., 2018), considering the onset of AMS after ascents and compliance of prophylactic medications, CDDPs was administered on the day before or prior to the ascents in this study. The subjects were thus administered with a regular dose of CDDPs (270 mg daily, three times a day (tid)) for two days prior to the ascents. The adaptability of the unacclimatized individuals was also evaluated to a hypoxial high-altitude environment through observing and analyzing a series of efficacy outcomes. The efficacy endpoints included AMS incidence upon rapid daily ascents to high-altitude regions, AMS severity within trial period, and time taken for the first incidence of AMS. Furthermore, comprehensive and single scores for AMS symptoms were recorded, and arterial oxygen saturation $\left(\mathrm{SaO}_{2}\right)$, heart rates, as well as blood pressures of the subjects were closely monitored. Adverse events in groups receiving prophylactic agents for AMS were also reported. This research could thus provide novel prophylactic and/or therapeutic measures for individuals who rapidly ascend to high-altitude regions and contribute to gaining perceptions of how CDDPs ameliorate hypoxia and its related syndromes.

\section{MATERIALS AND METHODS}

\section{Subjects}

A total number of 60 healthy male and female subjects aged 18 to 45 years, who were unacclimatized individuals residing in Beijing (average altitude of 46 meters), were recruited in this study. The effect size and the sample size in the design of this study could not be estimated, as there was no previously published reference research and a pilot study could not be carried out. The body mass index (BMI) of the subjects were between 19 and 24 and they all had ascended to high-altitude regions, situated in Tibet at an altitude above $3000 \mathrm{~m}$, for the first time. Through screening, a total of 58 subjects were found to be eligible, who were then randomly assigned to treatment group (group receiving CDDPs; $\mathrm{n}=29$ ) or placebo group (CON group; $n=29$ ). The exclusion criteria for the subjects were as follows: (1) women during menstruation, pregnancy, or lactation, (2) smokers or alcohol and/or drug abusers, (3) individuals with a definite history of drug allergies, (4) subjects treated with drugs (including TCM agents) within two weeks of the trial, (5) those with a history of blood donation or sampling within three months prior to recruitment (6), individuals with severe liver and/or kidney dysfunction, (7) cases with abnormal detection results for C-reactive protein (CRP) (i.e. exceeding normal limits), (8) subjects having clinically significant digestive system conditions, metal disorders, or metabolic conditions such as diabetes or hyperthyroidism, (9) subjects who had previously suffered from cardiovascular and/or cerebrovascular conditions, (10) individuals with fatigue syndrome (not due to hypoxia at high altitudes), (11) those having primary headaches, (12) subjects suffering from vomiting such as vomiting reflex, vomiting due to vestibular disorders, and/or functional neurological disorders (FNDs) caused by digestive disorders; (13) those having an allergic constitution, (14) subjects who had participated in other clinical trials within one month; and (15) individuals taking circulation-invigorating, immunity-boosting, or antioxidant drugs.

\section{Study design and randomization}

This study was a randomized, double-blind, and placebo-controlled trial. For this purpose, the subjects were 
randomly assigned to two cohorts, respectively receiving either 270mg CDDPs (27 mg/pill, product number: $160335)$ daily, tid, or an identical-looking placebo $(27 \mathrm{mg} /$ pill, product number: 160336$) 270 \mathrm{mg}$, tid. The processing technology of dripping pill coating (including CDDPs and placebos) as well as requirement of drug swallowing to the subjects both ensured that the active pills and placebos tasted the same. The subjects were then transported by train from the low altitude of $40 \mathrm{~m}$ to high altitude located above $3000 \mathrm{~m}$ in Tibet. Two days prior to ascending to the high altitude, they were administered with the given drugs in the train. Drug administrations on day 1 and day 2 on the train were counted as prophylactic treatments due to the stable environment of constant pressure and oxygen in the compartments. In the evening of day 3 , the subjects reached Lhasa (3658 m altitude) and stayed there for one day. Afterwards, they arrived at Dangxiong (4300 m altitude) by bus on day 5, and continued to take the medicines for other two days. The subjects ascended rapidly from $40 \mathrm{~m}$ to $4300 \mathrm{~m}$ on two separate occasions to an altitude of $3658 \mathrm{~m}$ at Lhasa on day 3 and an altitude of $4300 \mathrm{~m}$ at Dangxiong on day 5. During the total 11day study period, all the subjects took medicines for 7 days, and there was a subsequent three-day follow-up after administration for both groups to assess any delayed responses. The medication was taken orally or sublingually tid, 10 pills each time. Random numbers and allocation tables were also generated by biostatistics professionals using SAS 9.2 software. The random numbers were then distributed to the subjects in sealed envelopes to determine the treatment allocation (CDDPs or placebo). The subjects, investigators, and assessors knew nothing about the medication type (i.e. double-blind pattern) until the data analysis was finally completed. A detailed flowchart for this trial is given in Figure 1.

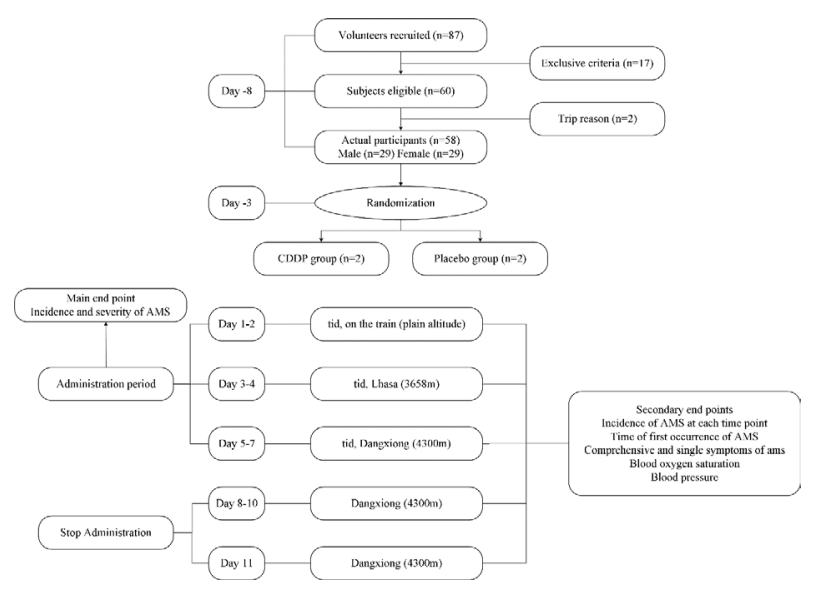

Fig. 1. Overview of trial protocol.

\section{Indicators and assessment methods}

The diagnostic criteria in this study were selected on the basis of the Lake Louise Scoring System (LLSS) as stipulated by the International Society for Mountain Medicine (ISMM), which is widely used to evaluate AMS incidence and severity (Wagner et al., 2012). The scoring system was composed of two parts: an AMS selfevaluation questionnaire and a clinical examination. The self-evaluation questionnaire was a 5-item, 4-point ordinal scale that included headaches; gastrointestinal symptoms such as anorexia, nausea, and/or vomiting; fatigue and/ or weakness; dizziness and/or light-headedness; and difficulty sleeping. The clinical assessment was also based on 3 items including change in mental status, ataxia (heelto-toe walking), and peripheral edema. Each item was graded on a scale ranging from 0 to 4 , with 0 indicating no symptoms at all and 1 to 4 implying mild, moderate, severe, and extremely severe symptoms; respectively. The subjects presenting self-evaluation questionnaire plus clinical examination scores amounting to 3 or above, including headaches were also diagnosed with AMS (Roach et al., 2018). Furthermore, severe AMS was defined as the presence of headaches and an LLSS $>5$ (Mandolesi et al., 2014). The AMS symptoms were additionally observed and evaluated twice a day during the research period (7:00-9:00 am and 19:00-21:00 pm).

$\mathrm{SaO}_{2}$, heart rates, and blood pressures were also monitored and recorded. $\mathrm{SaO}_{2}$ was measured by pulse oximetry (NONIN Onyx Vantage 9590, USA) using finger clips at baseline (just prior to drug administration on day 1) and twice a day (7:00-9:00 am and 19:00-21:00 pm) from day 2 to day 11. Heart rates and blood pressures (systolic/diastolic blood pressure, $\mathrm{mmHg}$ ) were still further monitored using an electronic sphygmomanometer (Omron, HEM-7132, China) twice daily at the same time at baseline (day 1) and for the following two days (days 1 and 2 on the train), and on days 3 to 11 of the high-altitude stay (7:00-9:00 am and 19:00-21:00 pm).

The primary endpoints were taken as the AMS incidence and severity during the whole trial period. The secondary endpoints included time of the first onset of AMS in a subject, comprehensive and single scores of AMS symptoms, as well as $\mathrm{SaO}_{2}$, heart rate, and blood pressure measurements, which were measured at baseline and during the whole study period (i.e. over the course of ascents and stays at high altitudes). Adverse events were ultimately recorded and reported by investigators using both scheduled and unscheduled visits.

Statistical analysis

All the statistical analyses were performed using SAS software package, version 9.2 (SAS Institute Inc., 
Cary, NC, USA). Two-sided P-values $<0.05$, were also considered to be statistically significant. Baseline, i.e., firstday, characteristics such as demographic information, vital signs, laboratory examination results, and medication taken by the subjects, were also expressed as mean \pm standard deviation $(S D)$ or counts (\%), as appropriate. As for the main endpoints, the Chi-square test (Fisher's exact test when appropriate) was utilized to compare AMS incidence and severity during drug administration (day 1 to day 8 ) and the whole observation period (day 1 to day 11) between groups. As for the secondary endpoints, the Chi-square test (Fisher's exact test whenever appropriate) was employed to test the difference of AMS incidence at each time point (day 1 to day 11) for the CDDPs and CON groups. The product limit method was also used to calculate the fourth percentile of the first AMS occurrence time and the bilateral $95 \%$ confidence interval (CI), as well as to obtain the Kaplan-Meier curve according to the incidence at each time point. Then, the log-rank test was employed to compare the time of the first AMS occurrence. The values of comprehensive and single-symptom scores at the baseline and on day 2 to day 11 between the groups were consequently compared using the independent samples t-test and the Wilcoxon rank-sum test; respectively. Finally, group comparisons of $\mathrm{SaO}_{2}$, heart rates, and blood pressures were performed using the independent samples t-test.

\section{RESULTS}

\section{Baseline characteristics}

All the eligible subjects were assessed in terms of demographic, physical, and systematic characteristics, vital signs, as well as AMS symptoms prior to prophylactic agent administration. A total of 58 subjects were also randomized, of which 4 subjects in the CON group withdrew, 2 subjects did not finish the trial owing to being intolerant to the AMS, and 2 subjects dropped out of the trial because of adverse events including hypertension and headaches, which were not related to the agent according to the investigators. In the group taking CDDPs, the subjects aged 21 to 44 years (26 on average); of which 11 subjects $(37.93 \%)$ were male and 18 cases $(62.07 \%)$ were female. In the CON group, the subjects aged 21 to 45 years (average 29), including $13(44.83 \%)$ males and $16(55.17 \%)$ females. According to a comparison of the baseline characteristics of both groups, the subjects were relatively balanced and comparable with regard to their age, levels of education, and general physical conditions (height, weight, heart rates, respiration, body temperature, and systolic and diastolic pressure). Their systematic (skin, neck, superficial lymph nodes, sensory functions, autonomic nerve functions, etc.) and clinical (anaphylaxis, drug allergy, and medical history) status were all normal (data not shown). No subjects were taking any other medications. The pregnancy tests for women of childbearing age were all negative. The proportions of nonsmokers and non-drinkers in the group receiving CDDPs and CON group were $28(96.55 \%)$ vs. $24(82.76 \%)$ and $27(93.10 \%)$ vs. 22 (75.86\%); respectively. The detailed baseline information is presented in Table I.

Table I. Baseline characteristics of the eligible subjects.

\begin{tabular}{lll}
\hline Characteristics & CDDPs (n=29) & CON (n=29) \\
\hline Gender (male:female) & $11(37.93 \%):$ & $13(44.83 \%):$ \\
& $18(62.07 \%)$ & $16(55.17 \%)$ \\
Age & $28.31 \pm 6.89$ & $30.31 \pm 7.06$ \\
Height $(\mathrm{cm})$ & $165.97 \pm 6.41$ & $167.03 \pm 8.31$ \\
Weight $(\mathrm{kg})$ & $61.22 \pm 6.99$ & $61.22 \pm 7.64$ \\
BMI $\left(\mathrm{kg} / \mathrm{m}^{2}\right)$ & $22.16 \pm 1.41$ & $21.90 \pm 1.73$ \\
Heart rate $(\mathrm{min})$ & $77.66 \pm 10.72$ & $76.76 \pm 11.27$ \\
Respiration & $18.76 \pm 2.50$ & $18.07 \pm 2.15$ \\
Body temperature $\left({ }^{\circ} \mathrm{C}\right)$ & $36.61 \pm 0.23$ & $36.51 \pm 0.28$ \\
SBP (mmHg) & $119.62 \pm 10.16$ & $119.45 \pm 13.76$ \\
DBP (mmHg) & $75.52 \pm 7.45$ & $76.17 \pm 7.94$ \\
SaO ${ }_{2}$ (\%) & $97.79 \pm 1.32$ & $97.86 \pm 1.36$ \\
Non-smokers & $28(96.55 \%)$ & $24(82.76 \%)$ \\
Non-drinkers & $27(93.10 \%)$ & $22(75.86 \%)$ \\
Chest X-ray (normal) & $22(75.86 \%)$ & $26(89.66 \%)$ \\
Comprehensive scores of & $0.55 \pm 1.12$ & $0.41 \pm 0.73$ \\
AMS symptoms & & \\
\hline
\end{tabular}

BMI, body mass index; SBP, systolic blood pressure; DBP, diastolic blood pressure; $\mathrm{SaO}_{2}$, arterial oxygen saturation. The continuous variable data are shown as mean $\pm \mathrm{SD}$.

\section{AMS incidence}

The incidence of AMS (LLSS $>3$ ) in the treatment group was the same as that in the CON group (both $8 / 29$ and $27.59 \%$, and $95 \%$ CIs: -23.00-23.00) during drug administration (day 1 to day 8). Also, there was no significant difference in the number of subjects who suffered from severe AMS (LLSS $>5$ ) between the groups. However, the incidence of severe AMS in the group receiving CDDPs was slightly lower than that in the CON group. If the occurrence of individual symptoms were regarded as actual cumulative incidence of AMS, the proportion of subjects affected with severe AMS (LLSS $>5$ ) in the treatment group was significantly lower (nearly 2.7-times) than that in the CON group $(22 / 634,3.4 \%$ vs. $56 / 602,9.3 \%, \mathrm{P}<0.01)$. Furthermore, 
there were 4 cases of withdrawal from the trial because of unsatisfactory efficacy. Thus, despite the seemingly insignificant reduction of AMS incidence in the group with the administration of CDDPs compared with that in the CON group during the administration period, CDDPs might be a useful adjuvant for decreasing incidence frequency and increasing resistance to AMS symptoms.

No significant differences were also observed between the treatment and CON groups in terms of the AMS incidence at each time point (all $\mathrm{P}>0.05$, Fig. 2). However, the AMS incidence in the group taking CDDPs was less than that in the CON group at almost every time point (except for the morning of day 4) during the whole study period as altitude increased. This trend became increasingly obvious as the administration time extended and reached a relatively stable state in the follow-up period. It is notable that, after rapid ascents to Lhasa (altitude 3658 $\mathrm{m}$ ) from low-altitude regions (altitude $43.71 \mathrm{~m}$ ) on day 3 , there were $3(n=29,10.34 \%)$ subjects experiencing AMS in the group receiving CDDPs, whereas there was one more case $(4 / 29,13.79 \%)$ in the CON group. However, only one case was observed in the group with CDDPs after arrival at Dangxiong (altitude $4300 \mathrm{~m}$ ), which was significantly lower than that in the CON group (3.45\% vs. $20.69 \%)$. Thus, the results indicated that prophylactic treatment with CDDPs could facilitate acclimatization and reduce the onset of AMS upon ascents to higher-altitude regions.

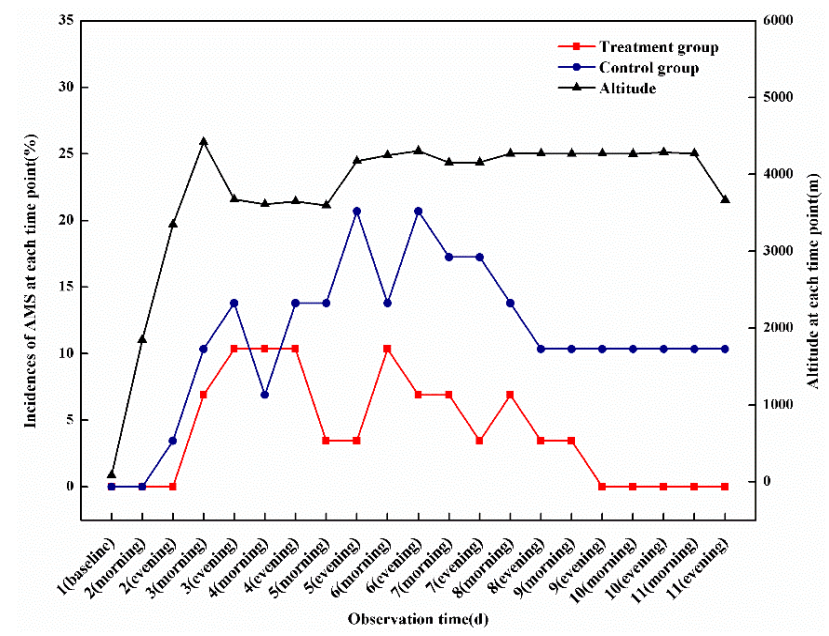

Fig. 2. Incidence of AMS at each time point.

\section{Effect of CDDPs on clinical symptoms of AMS}

During the whole observation period, the LLSS values for comprehensive AMS symptoms in the group taking CDDPs indicated lower occurrences than those in the CON group. It is noteworthy that in the evening of day 3 (arrival at Lhasa, altitude $3658 \mathrm{~m}$ ), the AMS symptoms for the CON group had clearly aggravated, with the average LLSS of 3.79. In contrast to those in the CON group, the subjects in the group with administration of CDDPs ascending to the same altitude had also experienced significant relief of AMS symptoms, with the average LLSS of $2.31(\mathrm{P}=0.0475)$, implying that drug administration prior to ascents could somewhat relieve the comprehensive symptoms related to AMS and decrease its severity. In the evening of day 5 , i.e. after continuing the ascent to Dangxiong (altitude $4300 \mathrm{~m}$ ), serious AMS symptoms reappeared among the subjects in the CON group, with an average LLSS of 3.17. Until day 9, the LLSS values exhibited a downtrend, suggesting that the clinical symptoms of AMS had slightly relieved in the subjects taking placebos. However, no obvious aggravation of the high-altitude symptoms (average LLSS=2.59) was observed in the group receiving CDDPs, even upon ascending further, and the LLSS values had begun to decline as early as day 6 . Towards the end of the observation period, the separation of the scores between the groups had also become increasingly significant, indicating that drug administration had enhanced the tolerance of the body and had also helped adapt to the high-altitude environment quickly. It is significant that the LLSS values in the group with CDDPs were again significantly lower than those in the CON group in the evening of day 10 and in the morning of day $11(\mathrm{P}=0.0367$ and $\mathrm{P}=0.0465$, respectively), which suggested that taking CDDPs had a more significant carryover effect on the subjects who had stayed at high altitudes for a long time. The LLSS values for the comprehensive clinical symptoms of AMS are given in Figure 3.

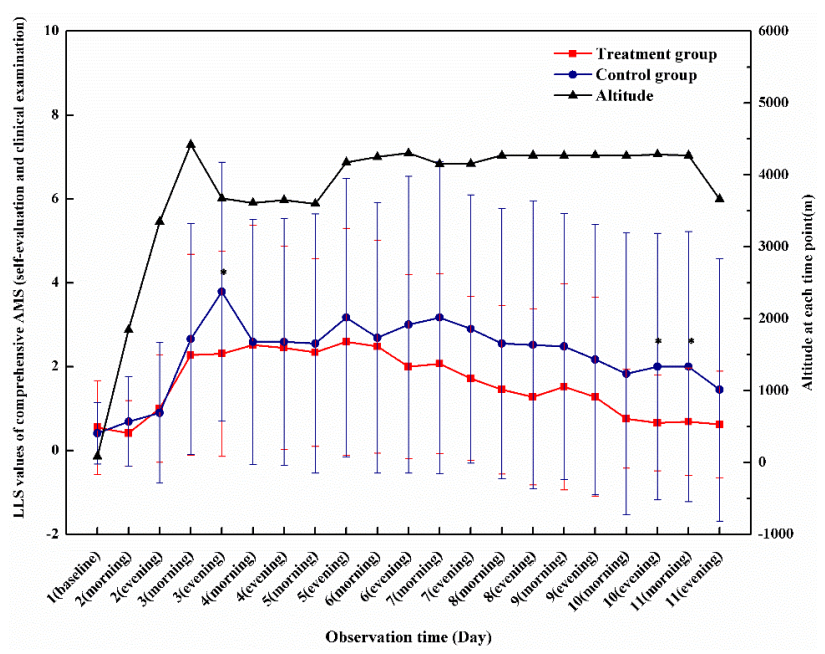

Fig. 3. LLSS values for comprehensive AMS symptoms for treatment and $\mathrm{CON}$ groups.

Asterisks indicate $\mathrm{P}<0.05$ between the treatment and CON groups at that observation time. 


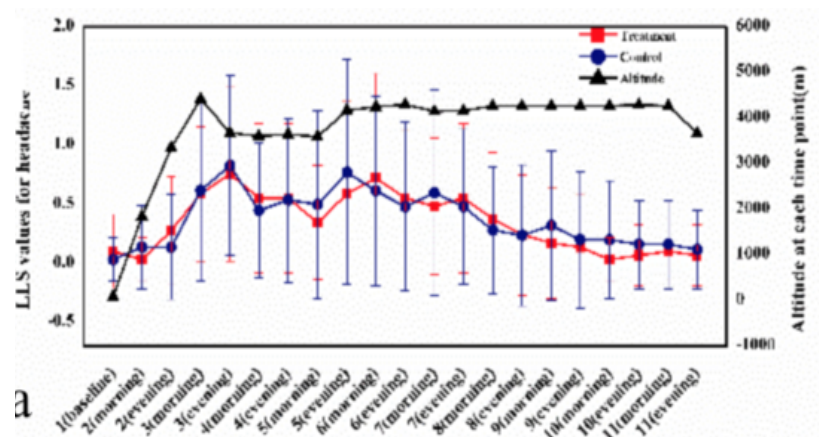

(Otservation timed()

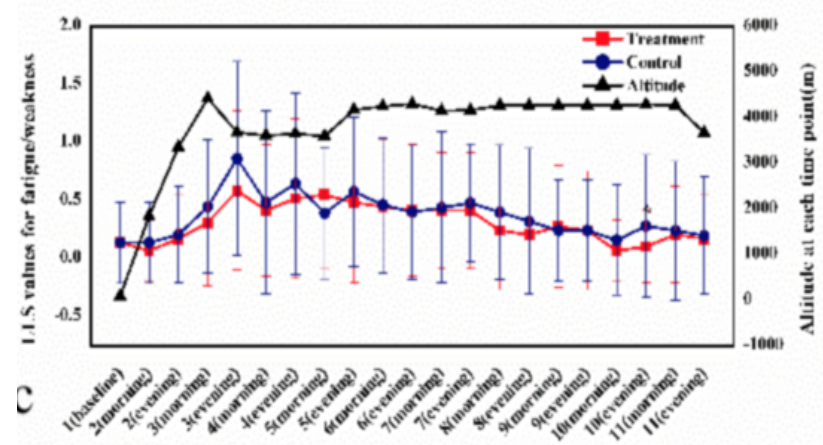

Observation time(d)

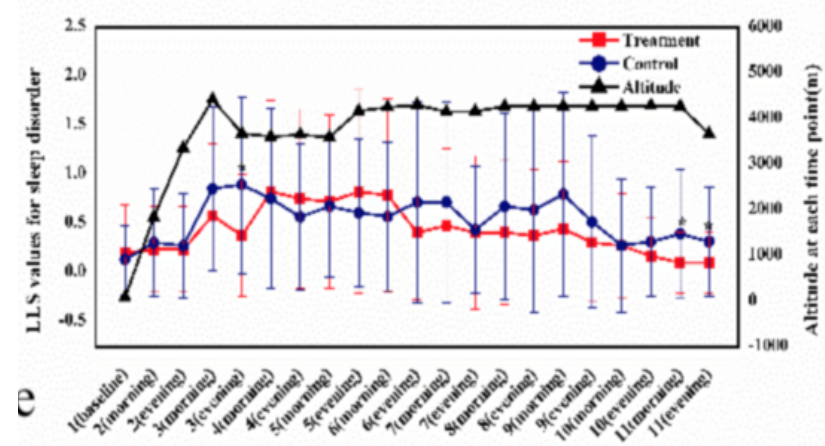

Ohservation time(d)

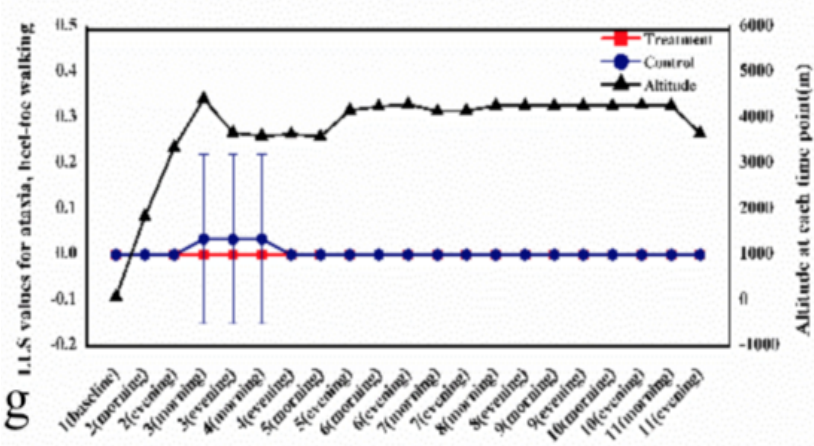

Observation timc(d)

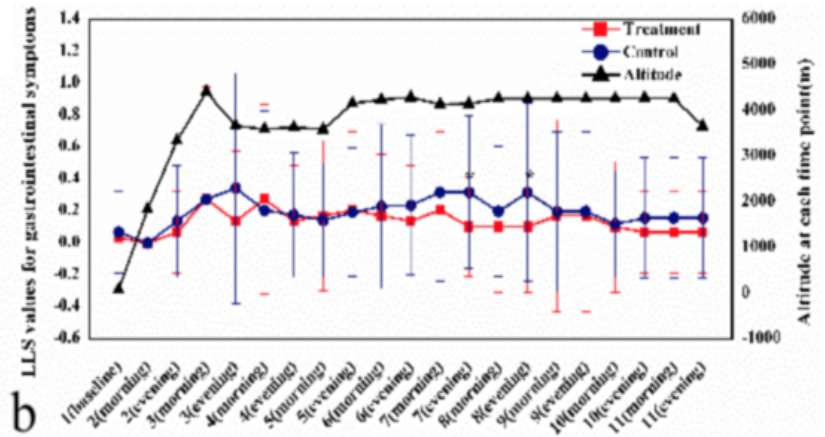

Observation time(d)

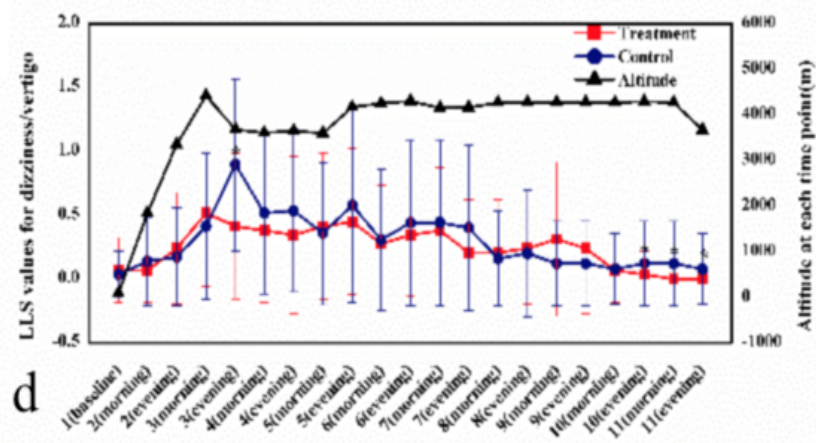

Obervation time(d)

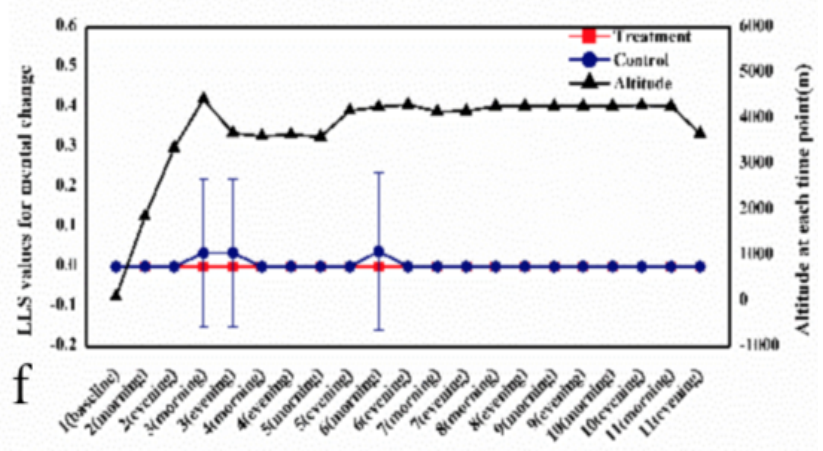

Observation time(d)

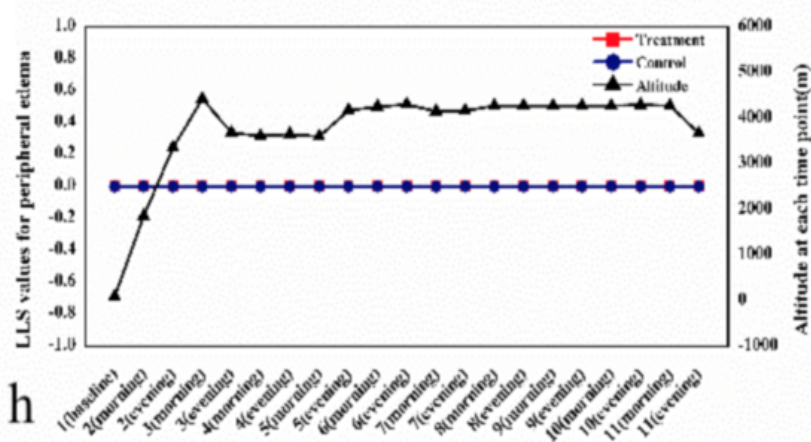

Observatien time(d)

Fig. 4. LLSS values for single AMS symptoms. (a) LLSS values for headache; (b) LLSS values for gastrointestinal symptoms; (c) LLSS values for fatigue/weakness; (d) LLSS values for dizziness/vertigo; (e) LLSS values for sleep disorder; (f) LLSS values for mental changes; (g) LLSS values for ataxia, heel-to-toe walking; (h) LLSS values for peripheral edema.

Asterisks indicate $\mathrm{P}<0.05$ between the treatment and $\mathrm{CON}$ groups at that observation time. 
The LLSS values of single clinical symptoms were also recorded to more accurately observe any AMS relief. As shown in Figure 4, there were no significant differences between the treatment and the CON groups for the LLSS of most single clinical symptoms. Nevertheless, the LLSS of the subjects taking CDDPs were clearly lower than those of the CON group after day 5 (pm). Additionally, the dizziness and/or light-headedness as well as sleep disorders in LLSS exhibited a generally similar trend to those for the comprehensive clinical symptoms, demonstrating that the subjects receiving CDDPs had developed significantly milder symptoms after rapid ascents to high-altitude regions $(\mathrm{P}=0.0070$ for dizziness/light-headedness; $\mathrm{P}=0.0106$ for sleep disorders, day 3 (p.m.)). No subjects in the treatment group had experienced any symptoms of mental change or ataxia during the whole study period, whereas there was one case of mental change and one case of ataxia in the CON group. No cases of peripheral edema had also appeared in either group. Thus, these results revealed that administration of CDDPs could help alleviate the clinical symptoms of AMS at high altitudes and improve adaptation to high-altitude environments.

\section{Time of first incidence of AMS after medication use}

As shown in Supplementary Information Table SI, the first case of AMS occurred on day 2 in the CON group and on day 3 i.e. after rapid ascents to Lhasa (altitude $3658 \mathrm{~m}$ ), in the group with CDDPs. Thus, the incidence of AMS for the treatment and CON groups was $13.79 \%$ vs. $17.86 \%$ on day 3 (pm). The median times of AMS occurrence were both longer than 8 days, with no significant differences between the study groups $(\mathrm{P}>0.05)$. However, upon staying at high altitudes (Dangxiong, altitude $4300 \mathrm{~m}$ ) for several days, especially on day 5 to day 7, the AMS incidence in the treatment group had a downtrend compared with that of the $\mathrm{CON}$ group.

\section{Effects of CDDPs on vital signs}

The two groups did not differ in terms of $\mathrm{SaO}_{2}$ during the medication period. However, once administration was stopped (i.e. for day 9 to day 11), the $\mathrm{SaO}_{2}$ values of the subjects in the group receiving CDDPs exhibited a downtrend in contrast to that in the $\mathrm{CON}$ group. Particularly, on Day 10 (am), the $\mathrm{SaO}_{2}$ value for the subjects taking CDDPs was significantly higher than that in the CON group ( $87.86 \%$ vs. $85.72 \%, \mathrm{P}=0.0388)$, suggesting that prophylactic drug administration could help relieve hypoxia-related symptoms. The complete $\mathrm{SaO}_{2}$ results are given in Supplementary Figure S1.

The heart rates in the subjects taking CDDPs and the placebo all remained within normal range throughout the study, and no obvious abnormality was observed in fluctuations. The average value of heart rates in the subjects receiving treatment was $79.03 / \mathrm{min}$ in the evening of day 2, which was significantly lower than those in the CON group (average $85.34 / \mathrm{min}, \mathrm{P}=0.0158$ ). In addition, there were no statistically significant differences between the two groups with regard to heart rates for the rest of time points (illustrated in Supplementary Fig. S2). The results for blood pressures were also similar to those for heart rates ( $\mathrm{P}>0.05$ for systolic pressures) except for the diastolic ones, whose average values in the group with CDDPs were significantly higher than those in the CON group on day 10 (am) $(88.38 \mathrm{mmHg}$ vs. $83.69 \mathrm{mmHg}, \mathrm{P}=0.0452)$ and day 11 (am) (89.66 $\mathrm{mmHg}$ vs. $84.76 \mathrm{mmHg}, \mathrm{P}=0.0382$ ). Nevertheless, both systolic and diastolic pressures of all the subjects were still in the normal range without any obvious abnormalities in fluctuations. The results for the blood pressures of all the subjects are shown in Supplementary Figure S3.

Table II. Adverse events in groups receiving CDDPs for AMS.

\begin{tabular}{lll}
\hline $\begin{array}{l}\text { Adverse event (Preferred } \\
\text { term) }\end{array}$ & $\begin{array}{l}\text { Group with } \\
\text { CDDPs }(\mathbf{n}=29)\end{array}$ & $\begin{array}{l}\text { CON group } \\
(\mathbf{n}=\mathbf{2 9})\end{array}$ \\
\hline Upper respiratory tract infection & $4(13.79 \%)$ & $4(13.79)$ \\
Amygdalitis & $1(3.45 \%)$ & $1(3.45)$ \\
Nausea & $0(0.00 \%)$ & $1(3.45 \%)$ \\
Non-infectious gingivitis & $1(3.45 \%)$ & $0(0.00 \%)$ \\
Abdominal discomfort & $1(3.45 \%)$ & $0(0.00 \%)$ \\
Vomiting & $0(0.00 \%)$ & $1(3.45 \%)$ \\
Headache & $0(0.00 \%)$ & $1(3.45 \%)$ \\
Backache & $1(3.45 \%)$ & $0(0.00 \%)$ \\
Hypertension & $0(0.00 \%)$ & $1(3.45 \%)$ \\
\hline
\end{tabular}

\section{Adverse events}

During the medication period, no CDDP-related effects were observed. The incidence of adverse events was also by $20.69 \%$. Only two subjects withdrew from the trial because of adverse events, including one case who exhibited a serious adverse event. The details are as follows: A 25-year-old female subject who had not previously ascended to high-altitude regions took regular medications on the way to Lhasa by train. On day 3 (am) she developed a serious headache and faintness, which impaired her ability to have meals, but showed no obvious coughing. So, administration of CDDPs was stopped and oxygen inhalation was administered to her. Her vital signs were also found to be basically normal, namely, her body temperature was $36.7^{\circ} \mathrm{C}$, her blood pressure was $105 / 75$ $\mathrm{mmHg}$, her respiration was $24 / \mathrm{min}$, and her $\mathrm{SaO}_{2}$ was by $88 \%$ prior to oxygen treatment. These symptoms were 
considered to be unrelated to the medication according to the investigators' judgments. Details on the adverse events during the research period are presented in Table II.

\section{DISCUSSION}

In this study, AMS-related symptoms as well as AMS severity were analyzed and assessed using the LLSS values of diagnostic criteria as formulated by the ISMM. The results indicated that administration of prophylactic CDDPs was effective for the relief of high-altitudeinduced hypoxemia, improvement of long-term tolerance to high altitudes, and acclimatization to mountainous environments. These effects were particularly notable in the relief of high-altitude-related faintness and sleep disorders on the day of rapid ascents to the plateau. All the subjects also underwent rapid acclimatization owing to the relief of fatigue through adequate rest and sleep on the train, with the AMS symptoms remaining in a relatively stable state and no obvious fluctuations in vital signs up to day 4 (am) and day 5 (am). However, the subjects in the CON group consistently experienced aggravated AMS symptoms upon rapidly ascending to high altitudes or continued ascents, with the symptoms that decreased until day 9. However, there was no obvious exacerbation of AMS symptoms for unacclimatized individuals taking CDDPs, even when rapidly ascending to high altitudes, especially in terms of altitude-related dizziness and sleep disorders. It is notable that, as early as the morning of day 6 , the LLSS values began to have downtrend until the end of the observation period, suggesting that the changes in AMS symptom scores in the CON group were in accordance with the characteristics of staircase acclimatization, and that prophylactic drug administration could help promote high-altitude acclimatization and enhance adaptability to hypoxic environments.

Altitude acclimatization is associated with physiological stress response that protects cells from damages caused by entering high-altitude hypoxic environments from low-altitude normoxic regions (Nussbaumer-Ochsner et al., 2012). The ability of lowaltitude residents to rapidly acclimatize to altitude exposure is accordingly dependent on physiological tolerance of the body to hypoxia (Oliver et al., 2012), altitude (higher altitudes are more difficult to acclimatize to) (Droma et al., 2006), ascent rates (Castellani et al., 2010), use of daily endurance exercises during ascents (Roach et al., 2000), and individual variability (Fulco et al., 2013). AMS and large decrements in endurance performance can also occur after rapid ascents to high-altitude environments owing to poor oxygen intake and difficulties in maintaining body homeostasis. Under these circumstances, physiological regulation mechanisms to accommodate the effects of hypoxia are triggered (Schoonman et al., 2008). Subsequently, the subjects will suffer mild AMS, generally lasting for several days, during which symptomatic relief takes about three days, and individual tolerance will be obtained in just less than one week (Dipasquale et al., 2015). This response is more obvious for subjects who have not taken prophylactic drugs, especially after rapid ascents. Therefore, acclimatization time in untreated subjects is relatively prolonged.

Pre-treatment with CDDPs helped to minimize hypoxia-induced AMS and correspondingly facilitated acclimatization. In this line, the results demonstrated that variations in heart rates and blood pressures in the treatment group were within the normal range and similar to those in the CON group $(\mathrm{P}>0.05)$. However, the $\mathrm{SaO}_{2}$ of the subjects taking CDDPs was significantly higher than those receiving placebos on day $10(\mathrm{P}<0.05)$. It should be noted that compensation for reduction in ambient oxygen is the foundation of altitude acclimatization, as the best strategy for AMS prevention, which allows individuals to retain their maximum physical and cognitive performance (Luks et al., 2019). Severely decreased $\mathrm{SaO}_{2}$ is also considered as an indicator of inadequate acclimatization and impending AMS, and elevated heart rates cannot be used to accurately predict impending AMS at altitudes of 4300 or $5300 \mathrm{~m}$ (Karinen et al., 2010). In this respect, Compte-Torrero et al. (2005) had observed that $\mathrm{SaO}_{2}$ in normal individuals had been affected to some extent by variations in altitudes. Normally, it was slightly lower after rapid ascents to a given altitude and increased gradually with acclimatization. The results of this study showed that taking CDDPs for several days prior to ascents could successfully maintain and somewhat enhance $\mathrm{SaO}_{2}$, thus minimizing AMS effects during periods at high altitudes. These results were significantly consistent with the assessments of clinical symptoms described above.

Numerous studies had further reported that prophylaxis with suitable drugs could prevent or alleviate AMS symptoms. Such drugs included acetazolamide, dexamethasone, nifedipine, sumatriptan, ginkgo biloba, and aspirin, which all had shown significant efficacy in comparison with a placebo (Davis and Hackett, 2017; Seupaul et al., 2012; Muhammad et al., 2019). Dexamethasone and acetazolamide are currently the most popular prophylactic agents for AMS prevention, and acetazolamide, approved by the Food and Drug Administration (FDA), is typically the first choice for preventing altitude-related sickness (Davis and Hackett, 2017). Moreover, Basnyat et al. had studied the effects of different doses of acetazolamide on altitude-related AMS and had found that the incidence rates of AMS at altitudes 
of $4280 \mathrm{~m}$ were $17 \%$ and $10 \%$ for subjects receiving 250 and $750 \mathrm{mg}$ doses of acetazolamide; respectively. However, the incidence had risen by $56 \%$ for $250 \mathrm{mg}$ acetazolamide and it had decreased to $17 \%$ with $750 \mathrm{mg}$ acetazolamide, indicating that altitude surge required an increase in the dosage of the medication (Basnyat et al., 2006). Regrettably, larger dosages of acetazolamide are associated with specific adverse effects in certain individuals, such as limb and facial numbness, lethargy, tinnitus, and gastrointestinal discomfort (Douglas et al., 1994). Consequently, it is prudent to make decisions regarding drug dosages following ascents to altitudes above $4000 \mathrm{~m}$. For acetazolamide-intolerant subjects, dexamethasone is also considered as a good alternative. As well, dexamethasone helps substantially lower the incidence of AMS at a ratio of $6.03(95 \% \mathrm{CI}, 2.23$ to 21.00) compared with a placebo (Tang et al., 2014). However, dexamethasone is a glucocorticoid and longterm treatment with this medication is likely to cause severe adrenal cortical dysfunction, low immunity, and other serious side effects (Vardy et al., 2006). Additionally, Dumont had observed that dexamethasone at dosages of 8-16 mg could prevent AMS above $4000 \mathrm{~m}$, but adverse reactions including gastrointestinal symptoms during treatment and depression after abrupt cassation, had occurred during the trial (Dumont et al., 2000). Thus, many chemical drugs are associated with specific adverse effects, and only high doses of acetazolamide are effective for AMS prevention. Accordingly, there is an urgent need to investigate extra alternatives. Among various TCM, Rhodiola crenulata is widely used to prevent AMS in the Himalayan areas and in Tibet. In the trials on AMS prevention using prophylactic herbal medication, Ginkgo biloba was the one mostly studied (Lee et al., 2013). For example, Chiu et al. had reflected on its efficacy in AMS prevention, however, the results had shown no significant differences in the incidence of AMS between $R$. crenulata extract and placebo groups (all 60.8\%; CI=0.69-1.52), denoting that $R$. crenulata was not effective in reducing AMS incidence or severity as compared with placebo (Chiu et al., 2013). This was similar to the reports in the present study. However, it was indicated that prophylactic CDDPs administration was effective for the relief of high-altitude-induced hypoxemia and improvement of long-term tolerance to high altitudes.

TCM is becoming increasing popular globally. For example, approximately $50 \%$ of residents in the United States use some form of alternative medicine to relieve specific symptoms (Wang et al., 2013). CDDPs are also efficacious for reducing the symptoms of coronary heart disease (CHD) and hastening the recovery of affected individuals without obvious flaws. This suggests that CDDPs have potential benefits for treating AMS. Additionally, there is ample evidence to support the potential of CDDPs for AMS prevention. For example, improving blood circulation is helpful for relieving highaltitude headaches as well as improving $\mathrm{SaO}_{2}$ (Wang et al., 2006). In this line, Jun et al. had found that rabbits taking CDDPs had exhibited decreased oxidative stress and inflammatory responses as well as improved cardiac functions compared with those in untreated animals. Naturally, this could be beneficial for lessening intracranial pressure, improving peripheral edema, and thus preventing individuals from developing more severe AMS-related manifestations such as HACE and HAPE.

Beside appropriate drugs, gradual ascents, keeping warm, avoiding fatigue, restricting alcohol, maintaining adequate hydration, and adhering to a high-carbohydrate diet were also beneficial to prevent AMS (Luo et al., 2013). To reach a better understanding of the AMS mechanisms, serum and urine samples could be collected and subjected to metabonomic analysis, which might help provide new insights into the effects of CDDPs in AMS treatment (Zou et al., 2015).

\section{CONCLUSION}

Administration of prophylactic CDDPs relieves faintness, sleep disorders, as well as other hypoxiainduced symptoms experienced at high altitudes, especially those occurring after rapid ascents, improving tolerance to hypoxial environments, and facilitating altitude acclimatization for unacclimatized individuals. Mitigating the clinical manifestations of AMS, treatment with CDDPs is also able to delay the onset of AMS and to reduce its incidence to a certain extent without any specific adverse effects. This research provides a relatively effective and safe alternative prophylactic AMS treatment for both unacclimatized individuals living at low altitudes and clinicians offering medication guidance. To achieve a more comprehensive understanding of the effects of CDDPs on humans, detailed pharmacokinetics and pharmacodynamics as well as mechanistic studies should be performed in the future.

\section{ACKNOWLEDGEMENTS}

We hereby gratefully express our thanks to Prof. Zhaoli Chen from the Institute of Health and Environmental Medicine, Key Laboratory of Risk Assessment and Control for Environment and Food Safety, for giving advice and reviewing this article. We also wish to extend our gratitude to all individuals for their assistance and contribution to 
this study.

Ethics approval and consent to participate

The study was conducted in accordance with the Declaration of Helsinki. It was also approved by the Ethics Committee of the Tibet Autonomous Region People's Hospital. As well, all the subjects provided written informed consent before recruitment and were allowed to withdraw the study at any time.

\section{Supplementary material}

All the data generated or analyzed during this study are included in this published article and its supplementary information files. Access the material online at: https:// dx.doi.org/10.17582/journal.pjz/20191204181230

\section{Statement of conflict of interests}

The authors have declared no conflict of interests.

\section{REFERENCES}

Basnyat, B., Gertsch, J.H., Holck, P.S., Johnson, E.W., Luks, A.M., Donham, B.P., Fleischman, R.J., Gowder, D.W., Hawksworth, J.S., Jensen, B.T., Kleiman, R.J., Loveridge, A.H., Lundeen, E.B., Newman, S.L., Noboa, J.A., Miegs, D.P., O’Beirne, K.A., Philpot, K.B., Schultz, M.N., Valente, M.C., Wiebers, M.R. and Swenson, E.R., 2006. Acetazolamide $125 \mathrm{mg} \mathrm{BD}$ is not significantly different from $375 \mathrm{mg} \mathrm{BD}$ in the prevention of acute mountain sickness: The prophylactic acetazolamide dosage comparison for efficacy (PACE) trial. High Alt. Med. Biol., 7: 17-27. https://doi.org/10.1089/ ham.2006.7.17

Carod-Artal, F.J., 2014. High-altitude headache and acute mountain sickness. Neurologia, 29: 533-540. https://doi.org/10.1016/j.nrleng.2012.04.021

Castellani, J.W., Muza, S.R., Cheuvront, S.N., Sils, I.V., Fulco, C.S., Kenefick, R.W., Beidleman, B.A. and Sawka, M.N., 2010. Effect of hypohydration and altitude exposure on aerobic exercise performance and acute mountain sickness. J. appl. Physiol., 109:1792-1800. https://doi.org/10.1152/ japplphysiol.00517.2010

Chiu, T.F., Chen, L.L., Su, D.H., Lo, H.Y., Chen, C.H., Wang, S.H.1. and Chen, W.L., 2013. Rhodiola crenulata extract for prevention of acute mountain sickness: A randomized, double-blind, placebocontrolled, crossover trial. BMC Complem. Altern. Med., 13: 298. https://doi.org/10.1186/1472-688213-298

Compte-Torrero, L., Botella, de Maglia, J., de DiegoDamiá, A., Gómez-Pérez, L., Ramírez-Galleymore,

P. and Perpiñá-Tordera, M., 2005. Changes in spirometric parameters and arterial oxygen saturation during a mountain ascent to over 3000 meters. Arch. Bronconeumol., 41 :547-552. https:// doi.org/10.1016/S1579-2129(06)60281-4

Davis, C., Hackett, P., 2017. Advances in the prevention and treatment of high altitude illness. Emerg. Med. Clin. N. Am., 35: 241-260. https://doi.org/10.1016/j. emc.2017.01.002

Dipasquale, D.M., Strangman, G.E., Harris, N.S. and Muza, S.R., 2015. Hypoxia, hypobaria, and exercise duration affect acute mountain sickness. Aerosp. Med. Hum. Perf., 86: 614-619. https://doi. org/10.3357/AMHP.4266.2015

Douglas Ried, L., Carter, K.A. and Ellsworth, A., 1994. Acetazolamide or dexamethasone for prevention of acute mountain sickness: a metaanalysis. J. Wildern. Med., 5: 34-48. https://doi. org/10.1580/0953-9859-5.1.34

Droma, Y., Hanaoka, M., Basnyat, B., Arjyal, A., Neupane, P., Pandit, A., Sharma, D. and Kubo, K., 2006. Symptoms of acute mountain sickness in Sherpas exposed to extremely high altitude. High Alt. Med. Biol., 7: 312-314. https://doi.org/10.1089/ ham.2006.7.312

Dumont, L., Mardirosoff, C., Tramèr, M.R., 2000. Efficacy and harm of pharmacological prevention of acute mountain sickness: Quantitative systematic review. Br. med. J., 321: 267-272. https://doi. org/10.1136/bmj.321.7256.267

Fulco, C.S., Beidleman, B.A. and Muza, S.R., 2013. Effectiveness of preacclimatization strategies for high-altitude exposure. Exerc. Sport Sci. Rev., 41: 55-63. https://doi.org/10.1097/ JES.0b013e31825eaa33

Hackett, P.H. and Roach, R.C., 2001. High-altitude illness. N. Engl. J. Med., 345: 107-114. https://doi. org/10.1056/NEJM200107123450206

Jafarian, S., Gorouhi, F., Salimi, S., Lotfi, J., 2010. Sumatriptan for prevention of acute mountain sickness: randomized clinical trial. Ann. Neurol., 62: 273-277. https://doi.org/10.1002/ana.21162

Jin, J., 2017. Acute mountain sickness. J. Am. med. Assoc., 318: 1840. https://doi.org/10.1001/ jama.2017.16077

Karinen, H.M., Peltonen, J.E., Kähönen, M. and Tikkanen, H.O., 2010. Prediction of acute mountain sickness by monitoring arterial oxygen saturation during ascent. High Alt. Med. Biol., 11: 325-332. https://doi.org/10.1089/ham.2009.1060

Kayser, B., Dumont, L., Lysakowski, C., Combescure, C., Haller, G. and Tramèr, M.R., 2012. Reappraisal 
of acetazolamide for the prevention of acute mountain sickness: A systematic review and metaanalysis. High Alt. Med. Biol., 13: 82-92. https:// doi.org/10.1089/ham.2011.1084

Lee, S.Y., Li, M.H., Shi, L.S., Chu, H., Ho, C.W., 2013. CTC: Rhodiola crenulata extract alleviates hypoxic pulmonary edema in rats. Evid-Based Compl. Alt. Med., 2013: 718739. https://doi. org/10.1155/2013/718739

Luks, A.M., Auerbach, P.S., Freer, L., Grissom, C.K., Keyes, L.E., McIntosh, S.E., Rodway, G.W., Schoene, R.B., Zafren, K. and Hackett, P.H., 2019. Wilderness medical society practice guidelines for the prevention and treatment of acute altitude illness: 2019 update. Wildern. Environ. Med., pili: S1080-6032(19)30090-0. https://doi.org/10.1016/j. wem.2019.04.006

Luks, A.M., Swenson, E.R. and Bärtsch, P., 2017. Acute high-altitude sickness. Eur. Respir. Rev., 26: pili: 160096. https://doi.org/10.1183/16000617.00962016

Luo, J., Song, W., Yang, G., Xu, H., Chen, K., 2015. Compound Danshen (Salvia miltiorrhiza) dripping pill for coronary heart disease: An overview of systematic reviews. Am. J. Chin. Med., 43: 25-43. https://doi.org/10.1142/S0192415X15500020

Luo, Y., Yang, X. and Gao, Y., 2013. Strategies for the prevention of acute mountain sickness and treatment for large groups making a rapid ascent in China. Int. J. Cardiol., 169: 97-100. https://doi. org/10.1016/j.ijcard.2013.08.059

Mandolesi, G., Avancini, G., Bartesaghi, M., Bernardi, E., Pomidori, L., Cogo, A., 2014. Long-term monitoring of oxygen saturation at altitude can be useful in predicting the subsequent development of moderate-to-severe acute mountain sickness. Wildern. Environ. Med., 25: 384-391. https://doi. org/10.1016/j.wem.2014.04.015

Métrailler, P., Greiser, J., Dietrich, G., Walter, D., Richon, J., Walliser, M., Wiget, U. and Sartori, C., 2019. Swiss mountain guides: Medical education, knowledge, and practice. High Alt. Med. Biol., Aug 2. https://doi.org/10.1089/ham.2018.0124

Muhammad, A.I., Nabila, R. and Ahmad, Q., 2019. Clinical trends of hepatic and renal profile in hyperthyroid subjects of Lahore, Pakistan. Punjab Univ. J. Zool., 34:1-7. http://dx.doi.org/10.17582/ journal.pujz/2019.34.1.1.7

Nussbaumer-Ochsner, Y., Ursprung, J., Siebenmann, C., Maggiorini, M. and Bloch, K.E., 2012. Effect of short-term acclimatization to high altitude on sleep and nocturnal breathing. Sleep, 35: 419-423. https://doi.org/10.5665/sleep.1708

O’Brien, K.A., Ling, S., Abbas, E., Dai, A., Zhang, J., Wang, W.C., Bensoussan, A., Luo, R., Guo, Z.X. and Komesaroff, P.A., 2011. A chinese herbal preparation containing radix salviae miltiorrhizae, radix notoginseng and borneolum syntheticum reduces circulating adhesion molecules. EvidBased Compl. Alt. Med., 2011: 790784. https://doi. org/10.1093/ecam/nen060

Oliver, S.J., Sanders, S.J., Williams, C.J., Smith, Z.A., Lloyd-Davies, E., Roberts, R., Arthur, C., Hardy, L., Macdonald, J.H., 2012. Physiological and psychological illness symptoms at high altitude and their relationship with acute mountain sickness: A prospective cohort study. J. Travel Med., 19: 210-219. https://doi.org/10.1111/j.17088305.2012.00609.x

Pandit, A., Karmacharya, P., Pathak, R., Giri, S., Aryal, M.R., 2014. Efficacy of NSAIDs for the prevention of acute mountain sickness: A systematic review and meta-analysis. J. Commun. Hosp. Int. Med. Perspect., 4: 24927. https://doi.org/10.3402/ jchimp.v4.24927

Roach, R.C., Hackett, P.H., Oelz, O., Bärtsch, P., Luks, A.M., MacInnis, M.J., Baillie, J.K., 2018. Lake Louise AMS Score Consensus Committee. The 2018 Lake Louise Acute Mountain Sickness Score. High Alt. Med. Biol., 19: 4-6.

Roach, R.C., Maes, D., Sandoval, D., Robergs, R.A., Icenogle, M., Hinghofer-Szalkay, H., Lium, D. and Loeppky, J.A., 2000. Exercise exacerbates acute mountain sickness at simulated high altitude. $J$. appl. Physiol., 88: 581-585. https://doi.org/10.1152/ jappl.2000.88.2.581

Schneider, M., Bernasch, D., Weymann, J., Holle, R. and Bartsch, P., 2002. Acute mountain sickness: influence of susceptibility, preexposure and ascent rate. Med.Sci. Sports Exerc., 34: 1886-1891. https:// doi.org/10.1097/00005768-200212000-00005

Schoonman, G.G., Sándor, P.S., Nirkko, A.C., Lange, T., Jaermann, T., Dydak, U., Kremer, C., Ferrari. M.D., Boesiger, P. and Baumgartner, R., 2008. Hypoxia-induced acute mountain sickness is associated with intracellular cerebral edema: A $3 \mathrm{~T}$ magnetic resonance imaging study. J. Cereb. Blood Flow Metab., 28: 198-206. https://doi.org/10.1038/ sj.jcbfm.9600513

Seupaul, R.A., Welch, J.L., Malka, S.T., Emmett, T.W., 2012. Pharmacologic prophylaxis for acute mountain sickness: a systematic shortcut review. Annls. Emerg. Med., 59: 307-317. https://doi. org/10.1016/j.annemergmed.2011.10.015 
Tang, E., Chen, Y. and Luo, Y., 2014. Dexamethasone for the prevention of acute mountain sickness: systematic review and meta-analysis. Int. $J$. Cardiol., 173: 133-138. https://doi.org/10.1016/j. ijcard.2014.03.019

Tsai, T.Y., Wang, S.H., Lee, Y.K., Su, Y.C., 2018. Ginkgo biloba extract for prevention of acute mountain sickness: a systematic review and meta-analysis of randomised controlled trials. Br. med. J., 8: e022005. https://doi.org/10.1136/ bmjopen-2018-022005

Vardy, J., Chiew, K.S., Galica, J., Pond, G.R., Tannock, I.F., 2006. Side effects associated with the use of dexamethasone for prophylaxis of delayed emesis after moderately emetogenic chemotherapy. $B r . J$. Cancer, 94: 1011-1015. https://doi.org/10.1038/ sj.bjc. 6603048

Wagner, D.R., Teramoto, M., Knott, J.R. and Fry, J.P., 2012. Comparison of scoring systems for assessment of acute mountain sickness. High Alt. Med. Biol., 13: 245-251. https://doi.org/10.1089/ ham.2012.1030

Wang, G., Wang, L., Xiong, Z.Y., Mao, B. and Li, T.Q., 2006. Compound salvia pellet, a traditional Chinese medicine, for the treatment of chronic stable angina pectoris compared with nitrates: A meta-analysis.
Med. Sci. Monit., 12: SR1-SR7.

Wang, J., Xiong, X., Xing, Y., Liu, Z., Jiang, W., Huang, J., Feng, B., 2013. Chinese herbal medicine for acute mountain sickness: A systematic review of randomized controlled trials. Evid-Based Compl. Alt. Med., 2013: 732562. https://doi. org $/ 10.1155 / 2013 / 732562$

Wilson, M.H., Newman, S., Imray, C.H., 2009. The cerebral effects of ascent to high altitudes. Lancet. Neurol., 8: 175-191. https://doi.org/10.1016/ S1474-4422(09)70014-6

Yang, R., Chang, L., Guo, B.Y., Wang, Y.W., Wang, Y.L., Jin, X., Liu, S.Y. and Li, Y.J., 2014. Compound danshen dripping pill pretreatment to prevent contrast-induced nephropathy in patients with acute coronary syndrome undergoing percutaneous coronary intervention. Evid-Based Compl. Alt. Med., 2014: 256268. https://doi. org $/ 10.1155 / 2014 / 256268$

Zou, H.M., Zhang, B., Xu, X.C., Su, J., Sun, Y.N., Xue, S., Wang, X.Y. and Qiu, M.F., 2015. Urinary metabolomic strategy to evaluate compound Danshen Dripping Pills for myocardial ischaemia in rats. J. Pharm. Biomed. Anal., 112: 98-105. https://doi.org/10.1016/j.jpba.2015.04.033 